\title{
Constructe a novel 5 hypoxia genes signature for cervical cancer
}

\author{
Yang Yang ${ }^{\dagger}$, Yaling $\mathrm{Li}^{\dagger}$, Ruiqun Qi and Lan Zhang* (D)
}

\begin{abstract}
Background: Hypoxia, which affects the development, metastasis and prognosis of cancer, represents a key feature of cancer. This study describe a hypoxia risk factor model, with predicting the prognosis of cervical cancer.

Methods: Based on hypoxia pathway related genes, we divided cervical cancer samples into high and low expression groups. A cox analysis was then performed. Genes from these cervical cancer samples showing a significant impact on OS were selected for cluster analysis to obtain two subtypes. The TPM dataset of TCGA was divided into training and validation sets. For the training set, a lasso analysis was conducted as based on cox analysis of meaningful genes and a risk factor model was constructed. The constructed model was verified in internal and external data sets. Finally, RT-PCR, immunohistochemistry were used to detect the expression of relative genes or proteins and functional assays were used to evaluate the biological function of signature genes.

Results: Two molecular subtypes were obtained, Cluster2 vs Cluster1.These subtypes were obtained by clustering with a total of 149 DEGs (Differential expressed genes) being in line with this standard, of which 27 were up-regulated and 122 were down-regulated. The five genes with lambda $=0.0571$ were selected to construct the model, the Ris $\mathrm{kScore}=\mathrm{AK}^{*} 0.042+\mathrm{HK}^{*} 0.021+\mathrm{P} 4 \mathrm{HA} 1^{*} 0.22+\mathrm{TGFB}{ }^{*} 0.1+\mathrm{VEGFA}{ }^{*} 0.077$. Further, in order to verify the signature, we used TCGA-test and GSE44001 chip datasets to test, and finally got a good risk prediction effect in those datasets. Moreover, the result of RT-PCR and immunohistochemistry demonstrated that AK4, HK2, P4HA1, TGFBI and VEGFA were all highly expressed in these cervical cancer tissue samples. The functional study shown that expression of AK4, HK2, P4HA1, TGFBI and VEGFA can regulate the proliferation, migration, and invasion ability of cervical cancer cells in vitro.

Conclusions: In summary, we developed a 5-gene signature prognostic hierarchical system based on the hypoxic pathway of cervical cancer, which is independent of clinical characteristics. And also conducted experimental verifications on these signature gene. Therefore, we propose that use of this classifier as a molecular diagnostic test can provide an effective means for evaluating the prognostic risk of cervical cancer patients, and provide potential targets for the treatment of cervical cancer patients.
\end{abstract}

Keywords: Hypoxia, Cervical cancer, Prognosis, Bioinformatics

*Correspondence: zhl851019@sina.com

†Yang Yang and Yaling Li are marked as co-first authors

Department of Dermatology, The First Hospital of China Medical University and National Joint Engineering Research Center

for Theranostics of Immunological Skin Diseases, The First Hospital of China Medical University and Key Laboratory of Immunodermatology, Ministry of Health and Ministry of Education, Shenyang 110001, China

\section{Background}

The most recent survey results indicate that cervical cancer ranks fourth in morbidity and mortality among women [1]. The findings that the incidence of this cancer varies among different countries and regions reveals that considerable geographic diversity exist. In addition, risk factors related to social and economic conditions are present within specific regions [2]. Persistent human papillomavirus (HPV) infection is a major cause of cervical 
cancer, with poor prognosis in developing countries [3, 4]. And the vaccine only targeted women before infection, will not help women who have been infected with HPV. CT and MRI are considered to be the most effective tools for use in the clinical evaluation of invasive cervical cancer [5], while PET-CT has become increasingly more common in preoperative examinations and diagnoses of metastatic cervical cancer. However, difficulties in detecting lymph node metastases and limited spatial resolutions diminish PET-CT's ability for detecting small lesions or early screening and diagnosis of primary lesions [6-8]. Cervical cancer is aggressive and often only identified in advanced stages. For women with metastatic or recurrent diseases, the overall prognosis remains poor [9]. As the prognosis of cervical cancer varies markedly as a function of patient's genetic heterogeneity, it is clear that the identification of effective genetic biomarkers would significantly enhance the capacity to predict the prognosis of this condition [10-12].

With the maturity of high-throughput sequencing technology, large-scale omics data generation is allowed $[13,14]$. Signatures of these hypoxia-related genes may explain cancer etiology and could hold diagnostic and prognostic value. However, cervical cancer has no hypoxia-associated prognostic signature has been established.

Hypoxia is a common cause of many malignant tumors and plays an important role in promoting the growth and metastasis of various tumors including cervical cancer $[15,16]$. Hypoxia activates several pathways leading to tumor epithelial-mesenchymal transition (EMT). EMT is a basic process of cancer invasion and involves different regulatory mechanisms depending on the types and subtypes of malignant tumors. Typically, cells undergoing this transformation lose many epithelial markers and ZO-1 expression, while mesenchymal markers are increased [17]. In addition, under hypoxic conditions, cancer cells exhibit adaptive metabolic changes, including the conversion of glucose to lactic acid and increased glucose uptake by promoting glucose transporters (GLUTs), known as the Warburg effect [18]. Results from a number of studies have shown that hypoxia is associated with the prognosis of many tumors [19-22]. Since hypoxia genes are closely related to the occurrence and development of cervical cancer, we attempt to develop a predictive model on the basis of hypoxic-related differential genes to provide accurate clinical guidance to cervical cancer patients. Although there have been some studies directed at developing prognostic models as based on gene signatures, few studies have focused on hypoxiarelated genes.

In our study, hypoxia genes were considered to be closely related to the progression of cervical cancer.
By analyzing the relationship between the expression of hypoxia-related genes and cervical cancer patients, we established a prognostic prediction model based on hypoxia genes, and evaluated the stability of the model through internal and external validation sets, which proved that the model is effective and possesses good predictive ability. This has not been reported in previous studies. In the future diagnosis and treatment of cervical cancer, genetic diagnosis and treatment will become effective means. By detection of the expression of model genes in cervical cancer tissues, and the conversion of model genes into risk scores, it is used to predict the prognosis of patients and has a clinical application value.

\section{Materials and method \\ Data download and preprocessing}

The gene expression profile and clinical follow-up information on cervical cancer patients were downloaded from TCGA and Gene Expression Omnibus (GEO) data (GSE44001). For processing of the RNA-Seq data of TCGA, while samples lacking clinical follow-up information were eliminated. Processing of the GEO dataset was performed in the following sequence, consisting of elimination of samples without clinical follow-up information survival time and status, transformation of the probe into a Gene Symbol, removal of probes corresponding to multiple genes and retention of maximal expression values with multiple Gene Symbols. For the hypoxia gene dataset, genes of the Hypoxia-related pathway (HALLMARK_HYPOXIA) were downloaded from MSigDB and 200 Hypoxia-related genes were sorted out.

\section{Cluster analysis of TCGA cervical cancer}

Initially, 200 genes related to the hypoxia pathway were downloaded from MSigDB(http://www.gsea-msigdb.org/ gsea/msigdb/index.jsp) for analysis. These samples were then divided into high and low expression groups according to the median of gene expression in the TCGA-TPM data of cervical cancer. A cox analysis, as based on overall survival (OS), was performed and genes with a $\mathrm{p}<0.05$ were screened. A forest map was then drawn using the forestplot package to illustrate results of the cox analysis. A Cluster analysis was performed on TCGA-TPM data of cervical cancer samples with genes selected that affected the OS. This Cluster analysis employed the $\mathrm{R}$ package ConsensusClusterPlus, with the parameters of the clustering algorithm being $\mathrm{k}$-means and the distance parameter euclidean. According to the CDF (Cummulative distribution function) and Delta Area, 2 represents the optimal $\mathrm{K}$ value. The OS-based survival curve of samples in the Cluster was then drawn. 
Identification of differentially expressed genes (DEGs) and functional enrichment analysis

The limma package was used to analyze the two subtypes, as obtained from clustering. The principle for screening differential genes was abs $(\operatorname{logFC})>1.2$ and an adj. of $\mathrm{p}<0.05$.Genes showing differential expressions were identified to construct a volcanic map with ggplot2 package. With use of the clusterprofiler package, GO (Gene Ontology) and KEGG (Kyoto Encyclopedia of Genes and Genomes) pathway analyses were performed on DEGs.

\section{Grouping TCGA cervical cancer dataset}

The 257 samples in the TCGA data set were divided into a training and test set, according to the proportion of training set:test set $=7: 3$. Finally, training and test set samples were displayed, and the chi-square test was used to verify the rational for the grouping.

\section{Construction of prognostic model based on genes related to the hypoxia pathway}

A cox analysis was initially performed on the training set data based on genes related to the hypoxia pathway. The samples were divided into high and low expression groups based on the median of gene expression and the effects of high and low expression on prognosis were analyzed, with genes affecting OS being screened $(\mathrm{p}<0.05)$. Then, as based on the above hypoxia-related pathway genes demonstrating an impact on OS, the $\mathrm{R}$ software package glmnet, was used to perform a lasso cox regression analysis on data of the test set samples. The trajectory of independent variables reveals that with a gradual decrease in lambda, the number of independent variable coefficients tending to 0 increases gradually. A tenfold cross validation was used to construct the model and analyze confidence intervals under each lambda. With a lambda $=0.0571$, the model is optimally effective, therefore genes at this point were used to construct the model. Survival curves were then drawn for the training set of the genes used for modeling.

\section{Construction, evaluation and verification of the prognostic model}

Within the training set, RiskScore of each sample were calculated according to expression levels of the sample and the RiskScore distribution was drawn for the sample. Next, the ROC analysis for the prognostic classification of RiskScore was performed using the R package, pROC. As based on the calculated median of RiskScore, samples were divided into high and low risk groups and survival curves were plotted. As a means to assess the robustness of this model, the same model and coefficient as the training set was used for the internal validation set and all data sets of TCGA. The predictive effect of RiskScore between high and low risk groups were evaluated. Next, the same coefficient as the training set for the external validation data set GSE44001 were used. Again, the predictive effect of RiskScore on the prognosis between the high and low risk groups were performed.

\section{Risk assessment of clinical subtypes}

A boxplot was then used to show the distribution of RiskScore in Grade3/4 and Grade1/2samples. Clinical subtypes such as Grade (1/2), Grade (3/4), Stages (I/II), Stages (III/IV), Age $(>65)$ and Age $(\leq 65)$ were grouped as based on the expression of Risk score to evaluate the prognosis of high and low risk groups.

\section{Univariate and multivariate cox analyses}

Univariate and multivariate COX regressions were used to analyze relations among risk scores, other variables and clinical prognosis of patients.

\section{RiskScore and clinical features for construction of the nomogram}

Based on results of the univariate and multivariate analyses a nomogram model was constructed with the clinical features Age, Stage and RiskScore, and as a means to generate a calibration curve to evaluate the prediction accuracy of the model. In addition, the DCA (Decision Curve Analysis) was used to assess the reliability of the model.

\section{Tissue samples}

Cervical cancer samples and adjacent non-tumor tissues were collected from 10 patients (all $>16$ years of age), immediately placed in liquid nitrogen and preserved at $-80^{\circ} \mathrm{C}$. Cervical cancer samples including 7 cervical squamous cell carcinomas and 3 cervical adenocarcinoma. None of the patients received any preoperative anti-tumor therapies. All patients and their families were fully informed as to the purpose of the study and informed consent was obtained from all participants. This study was approved by the Ethics Committee of the First Hospital of China Medical University (2016-207-4).

\section{Cell lines and culture conditions}

Cervical cancer cells, Hela, were purchased from the Chinese Academy of Medical Sciences and the CAMS \& PUMC Medical College (Beijing, China). Cell lines were cultured in 1640 medium supplemented with $10 \%$ fetal bovine serum and 100 units/ml penicillin at $37{ }^{\circ} \mathrm{C}$ in a humidified $5 \% \mathrm{CO}_{2}$ incubator.

\section{RNA isolation and RT-PCR analysis}

RNA extraction from tissues was performed using the TRIzol reagent (Invitrogen, Carlsbad, CA, USA). RNA 
was reverse-transcribed into cDNA with use of the QuantiTect Reverse Transcription Kit (QIAGEN, Valencia, CA, USA). Real-time PCR analyses were quantified by SYBR-Green (Takara, Otsu, Shiga, Japan) and levels were normalized to GAPDH levels. Sequences of upstream and downstream primers are contained in Additional file 11: S6.xls.

\section{Immunohistochemistry}

Cervical cancer samples were fixed in $10 \%$ formalin, embedded in paraffin and processed as $5-\mu \mathrm{m}$ sequential sections. Samples were dewaxed with ethanol and blocked to inhibit endogenous peroxidase activity. They were then heated in a microwave to retrieve antigens, cooled to room temperature and then blocked by incubation in goat serum for $30 \mathrm{~min}$ at $37 \circ \mathrm{C}$. Samples were incubated overnight at $4{ }^{\circ} \mathrm{C}$ with rabbit anti-AK4 (PA560,104), anti-HK2 (PA5-83,021), anti-P4HA1 (PA518,669), anti-TGFBI (PA5-82,358), and anti-VEGFA (PA5-85,171) (Thermo Fisher Scientific MA, USA; 1:1, 200), followed by incubation with horseradish peroxidase-coupled goat anti-rabbit secondary antibody at 37 ${ }^{\circ} \mathrm{C}$ for $30 \mathrm{~min}$ and then stained using $3,3^{\prime}$-diaminobenzidine. Cell nuclei were stained blue with use of hematoxylin. Sections were then dehydrated, cleared with xylene, and mounted. AK4, HK2, P4HA1, TGFBI and VEGFA expressions were determined with use of IHC (Immunohistochemistry) using a streptavidin peroxidase method, with adjacent tissues as controls. The experimental procedure was performed according to the manufacturers' instructions. TheImage-ProPlus 6.0 Software (MediaCybernetics, USA) was used to analyze protein expressions and perform statistics on the results obtained from immunohistochemistry.

\section{RNA interference}

SiRNAs against AK4 (NO. HSS164266), HK2 (NO. HSS179238), P4HA1 (NO. HSS107536), TGFBI (NO. HSS186313), VEGFA (NO.HSS111274) and negative control siRNA sequences from Thermo Fisher Scientific (MA, USA). The transfection was performed using Lipofectamine 3000 reagent (Invitrogen, Shanghai, China) according to the manufacturers' instructions. Cells were harvested for cytological analysis at $48 \mathrm{~h}$ post-transduction.

\section{Cell migration and invasion assays}

Cells were harvested, resuspended in serum-free media and placed in the upper chamber of a Transwell membrane filter (Corning, NY, USA) for migration assays or in the upper chamber of a Transwell membrane filter coated with Matrigel (Corning) for invasion assays. Culture medium with $10 \%$ FBS was added to the lower compartment of the chamber to serve as a chemoattractant. After $24 \mathrm{~h}$ of incubation, cells were stained with methanol and $0.1 \%$ crystal violet, imaged and counted using an Olympus microscope (Tokyo, Japan).

\section{Generation of single-cell-derived cellular clones}

Cell densites were adjusted to $5 \times 10^{3} / \mathrm{ml}$ and cell suspensions were contained in $100 \mu \mathrm{l}$ per well in the 6-well plates with $2 \mathrm{ml}$ of DMEM supplemented with 10\% FBS and $100 \mathrm{U} / \mathrm{ml}$ penicillin. Single-cell-derived clones were grown for 7 days. Cultures were pre-cooled three times with PBS, the methanol was fixed for 15 min, crystal violet dye was applied for $20 \mathrm{~min}$ and the water was rinsed and air dried. The number of visible clones was visually counted and a clone formation rate was calculated: clone formation rate $(\%)=$ (clone number/number of inoculated cells) $\times 100 \%$. This procedure was replicated three times.

\section{Statistical analysis}

All data were analyzed using the SPSS 21.0 statistical software program (IBM Corporation, Armonk, NY, USA). Graphs were generated with GraphPad Prism 8.0 Software (GraphPad Software, Inc., San Diego, CA). Student's t-tests were used. For t-tests, a two-tailed $p<0.05$ was required for results to be considered as statistically significant.

\section{Results}

\section{Sample information statistics}

The gene expression profile and clinical follow-up information on cervical cancer patients were downloaded from TCGA and GEO data (GSE44001). A total of 557 samples were obtained, including 257 TCGA and 300 GSE44001 samples. The steps to establish the model of prognostic characteristics are as Fig. 1. The preprocessed cervical cancer dataset is contained in Table 1.

For describing a hypoxia cervical cancer risk factor model, 200 genes related to the hypoxia pathway downloaded from MSigDB for analysis. The TCGA-TPM data of cervical cancer samples were divided into high and low expression groups according to the median of gene expression and a cox analysis as based on OS (the cox analysis results of all hypoxia pathway-related genes are shown in Additional file 1: S1.csv) was conducted. Genes with a $\mathrm{p}<0.05$ were screened and a forest plot package was used to draw the forest map for display of the cox analysis results (Fig. 2a, b). Among these genes, S100A4, NCAN, LDHC, ISG20 and GCNT2 $(\mathrm{HR}<1, \mathrm{p}<0.05)$ were identified. VEGFA, TGFBI, STC1, SLC2A1, SERPINE1, PPFIA4, PLAUR, PGM2, PGK1, PFKP, PFKFB3, PDK1, P4HA2, P4HA1, NFIL3, NDRG1, MIF, LDHA, JUN, IER3, HK2, GBE1, GAPDH, EXT1, ERO1A, ENO1, 


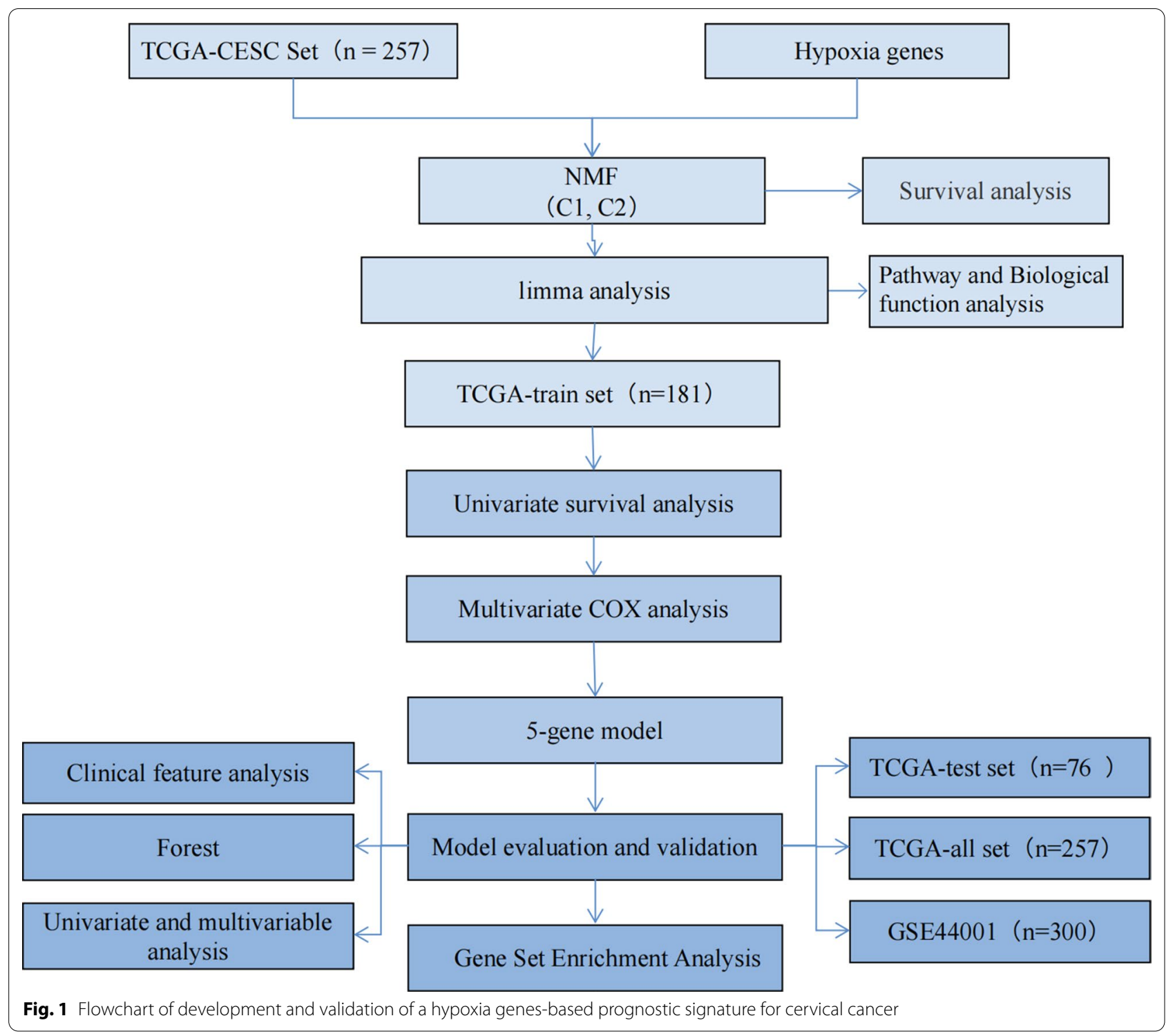

EGFR, EFNA3, EFNA1, DDIT4, CAVIN3, ANGPTL4, AK4 and ADM $(\mathrm{HR}>1, \mathrm{p}<0.05)$ were found to have characteristics of high expression and poor prognosis. The TCGA-TPM data of cervical cancer samples were analyzed with the above genes that affected the OS. According to the CDF and Delta Area, a value of 2 indicates the optimal K (Fig. 2c, d). Results from plots of OSbased survival curves of the samples in the two Clusters revealed that the capacity for prognosis from samples in Cluster2 was significantly increased over that in Cluster1 (Fig. 2e, p <0.0001).

\section{Differential genes analysis of two molecular subtypes}

The limma package was used for the difference analysis of the two subtypes, Cluster2 vs Cluster1. These subtypes were obtained by clustering with a total of 149 DEGs being in line with this standard, of which 27 were upregulated and 122 were down-regulated. Differentially expressed genes were displayed with use of ggplot2 package (Additional file 2: Fig. S1a, Additional file 3: S2.csv). Heat map of was drawn for the display of these results (Additional file 2: Fig. S1b).

\section{KEGG pathway and Go enrichment analysis}

Furthermore, we performed KEGG pathway and GO functional enrichment analyses on differentially expressed genes. With use of the clusterprofiler package, 27 up-regulated and 122 down-regulated genes were analyzed to determine their biological functions and pathways. A Barplot was used to display the eight enriched 
Table 1 Clinical information of cervical cancer cohort

\begin{tabular}{lll}
\hline Clinical features & TCGA-CESC & GSE44001 \\
\hline OS & & DFS \\
0 & 188 & 262 \\
1 & 69 & 38 \\
Stage & & \\
Stage I & 138 & \\
Stage II & 58 & \\
Stage III & 36 & \\
Stage IV & 19 & \\
Grade & & \\
G1 & 16 & \\
G2 & 116 & \\
G3 & 99 & \\
G4 & 1 & \\
Age & & \\
$\leq 65$ & 229 & \\
$>65$ & 28 & \\
\hline
\end{tabular}

KEGG pathway (Additional file 4: Fig. S2a), Biological Process (Additional file 4: Fig. S2b) and Molecular Function (Additional file 4: Fig. S2c), with a detailed analysis of these results presented in Additional file 5: S3.csv and Additional file 6: S4.csv. The KEGG pathway includes ECM-receptor interaction, focal adhesion, IL-17 signaling pathway, PI3K-Akt signaling pathway and the TGFbeta signaling pathway. The Biological Process terms includes extracellular matrix organization, epithelial cell proliferation, cell-matrix adhesion, cell adhesion mediated by integrin, positive regulation of epithelial to mesenchymal transition, regulation of epithelial to mesenchymal transition. The Molecular Function terms includes extracellular matrix structural constituent, receptor ligand activity, cytokine activity, extracellular matrix binding, epidermal growth factor receptor binding, growth factor binding, chemokine activity and interleukin-1 receptor binding.

\section{Grouping TCGA cervical cancer dataset}

For Construction of a prognostic risk model, the 257 samples of the TCGA cervical data set were divided into training and test set according to the proportion of

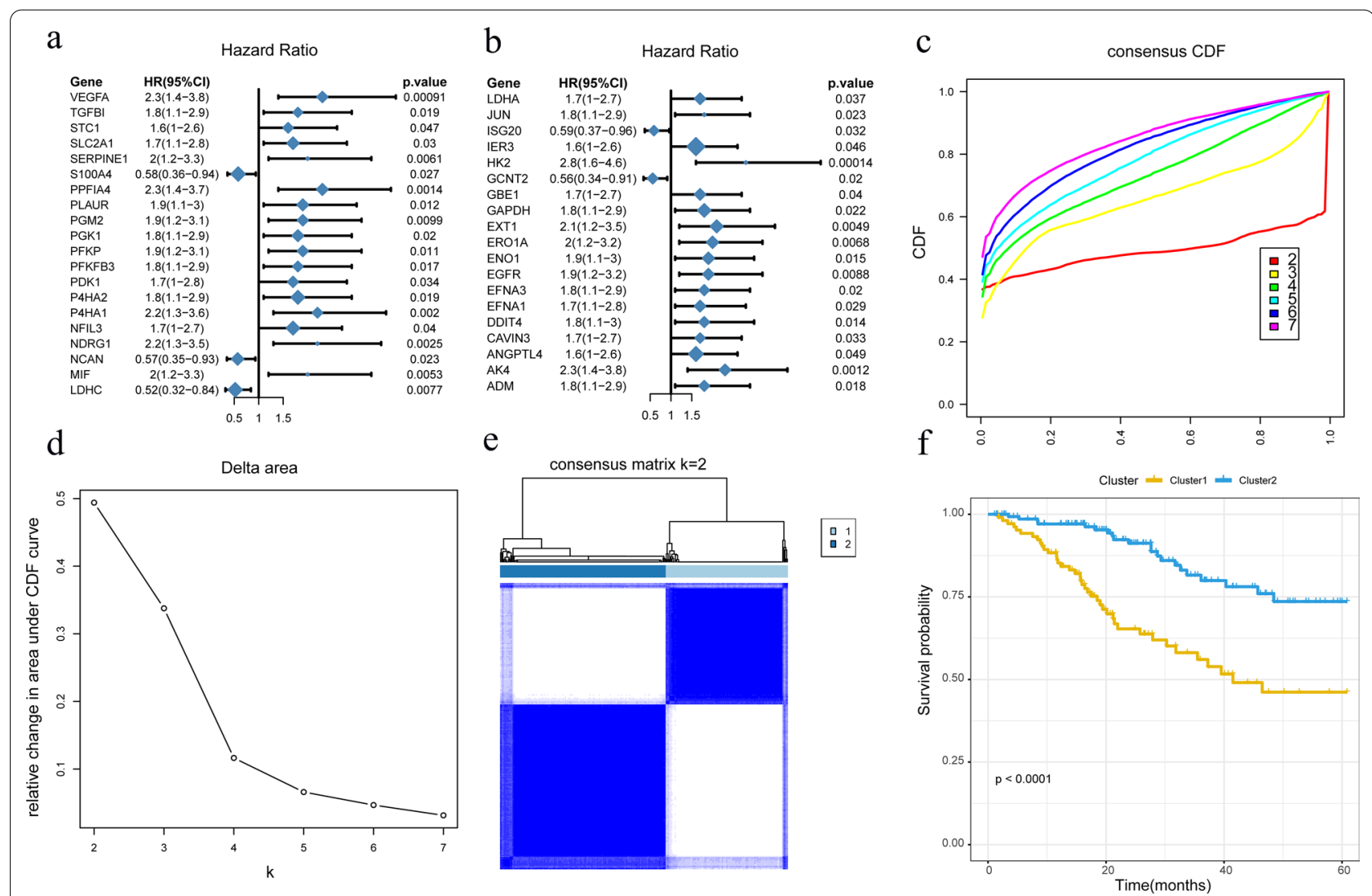

Fig. 2 Cox analysis of hypoxia pathway genes. a Results 1. b Results 2. c Cumulative distribution map of clustering consistency. $\mathbf{d}$ Clustering Delta Area map. e Display of clustering results of hypoxia pathway genes. f OS prognosis survival curves of different Clusters 
training set: test set $=7: 3$. The final training and test set samples are displayed and the chi-square test was used to indicate that the grouping was unbiased (Table 2, $\mathrm{p}>0.05)$.

\section{Generation of the prognostic model as based on genes related to the hypoxia pathway}

Training set datas were used for further analyse, Univariate Cox analysis was performed on differential genes among molecular subtypes, and Lasso regression was

Table 2 Grouping information of training set and validation set

\begin{tabular}{llll}
\hline Clinical features & TCGA-test & TCGA-train & $\mathbf{p}$ \\
\hline OS & & & \\
0 & 53 & 135 & 0.518 \\
1 & 23 & 46 & \\
Grade & & & 0.215 \\
$1 / 2$ & 34 & 98 & \\
$3 / 4$ & 42 & 83 & 0.799 \\
Stage & & & \\
I/II & 61 & 141 & 0.923 \\
III/IV & 15 & 40 & \\
Age & & & \\
$>65$ & 9 & 19 & \\
$\leq 65$ & 67 & 162 & \\
\hline
\end{tabular}

used to reduce the gene numbers in the risk model. As the lambda value decreases, the number of independent variable coefficients trending toward 0 increases (Fig. 3a). As indicated in Fig. 3b, the model was optimal when lambda $=0.0571$. Therefore, the five genes with lambda $=0.0571$ were selected to construct the model, the $\quad$ RiskScore $=$ AK $4 * 0.042+$ HK2 $* 0.021+$ P4HA1 $* 0.22$ + TGFBI*0.1 + VEGFA*0.077.

For the five genes used in the model (Fig. 3c-g), survival curves were drawn in the training set. The results show that all 5 genes can significantly distinguish the prognosis of high and low groups.

\section{Construction, evaluation and validation of the risk model}

To determine the robustness of the model, we used the TCGA training set, test set, all TCGA datasets and GSE44001 datasets to calculate RiskScore and drew the RiskScore distribution. In the training set, risk scores of each sample were calculated according to the expression level of the sample with an illustration of the RiskScore distribution of samples shown in Fig. 4a. As is evident from this figure, High expression of AK4, HK2, P4HA1, TGFBI and VEGFA were associated with a high risk, which indicates a risk factor. ROC analysis of riskScore prognostic classification using the $\mathrm{R}$ software package, pROC, revealed that the AUC (Area Under the Curve) of the model was greater than 0.68 at 1,3 and 5 years (Fig. 4b). According to the median of RiskScore, cervical

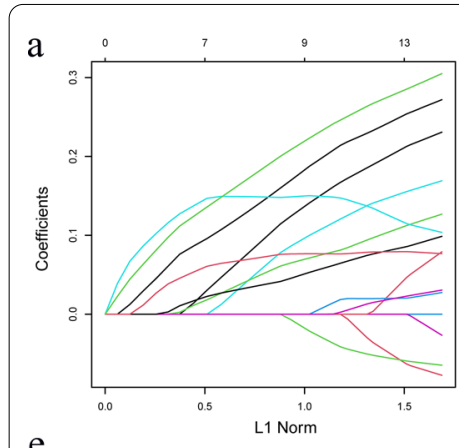

e

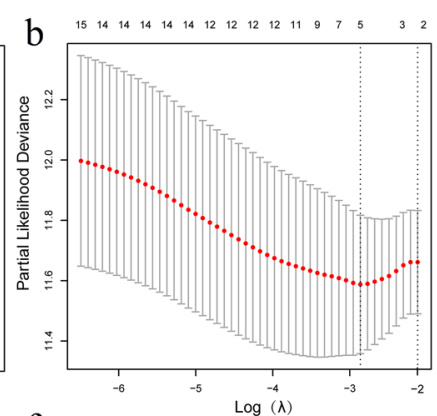

f

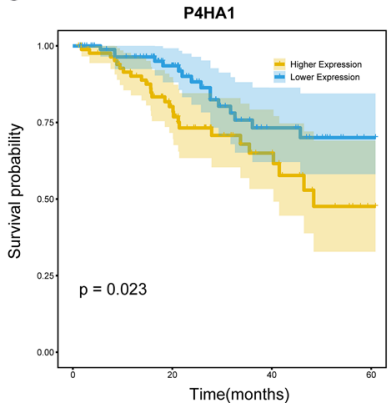

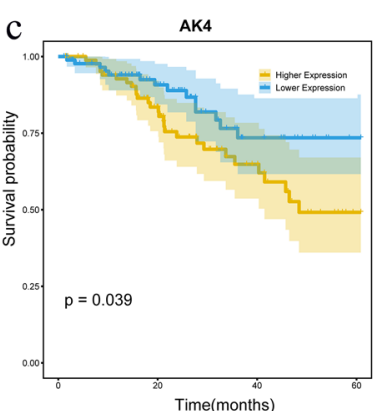

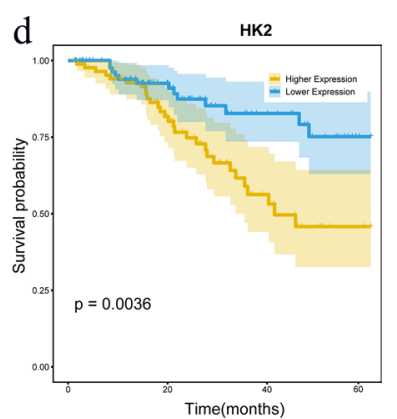

$\mathrm{g}$

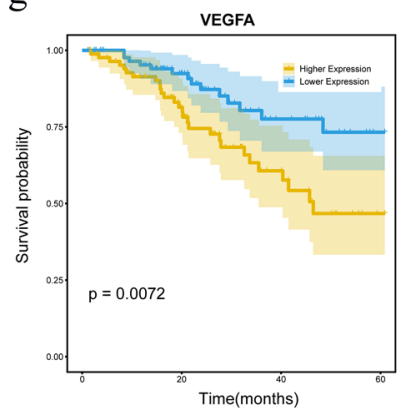

Fig. 3 a Changes in trajectory for each independent variable with the horizontal axis representing the $L 1$ norm and vertical axis the coefficient of the independent variable. $\mathbf{b}$ Confidence intervals under each lambda. c-g Survival analysis curves for the five prognostic model genes 

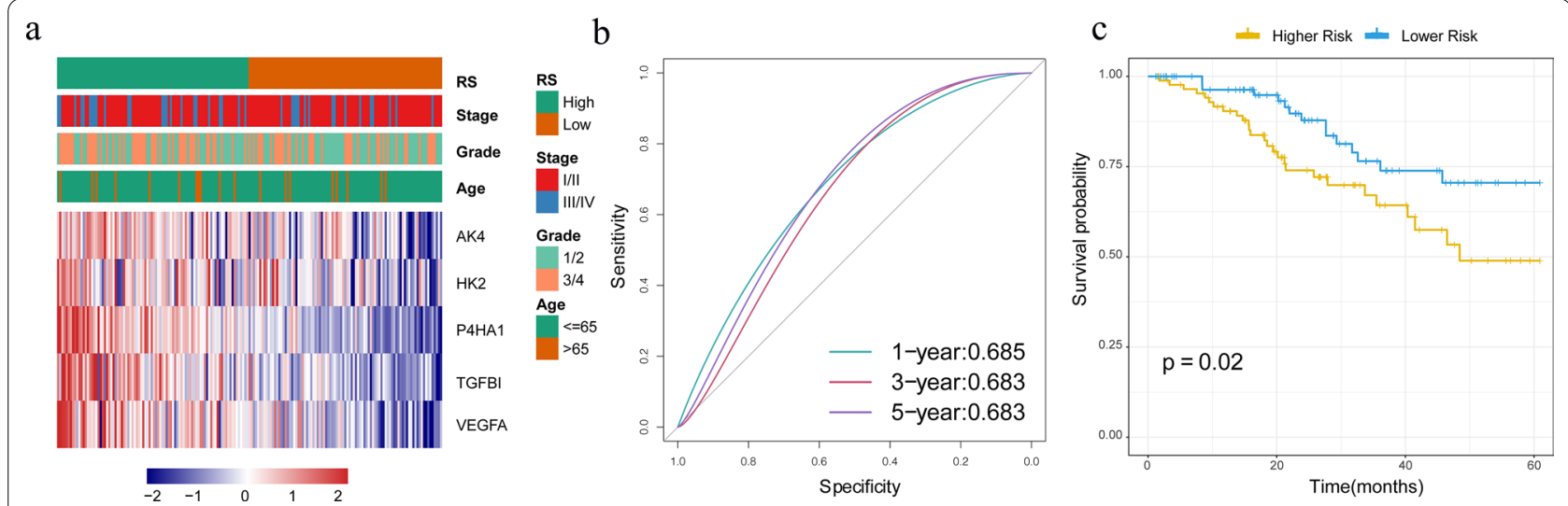

Fig. 4 a Expression and clinical features of the 5 prognostic genes in the high and low risk groups of the training set. $\mathbf{b}$ ROC curves and AUC of RiskScore classifications. c KM survival curve distribution of the RiskScore in the training set

cancer samples were divided into High RiskScore group and Low RiskScore group, and the survival curve was plotted by KM analysis. It can be seen that the prognosis of Low RiskScore risk group was significantly better than that of High RiskScore group (Fig. 4c).

In order to determine the robustness of the model, the same coefficients as obtained with the training set for the internal validation set and all data sets of TCGA were used. With this model, The RiskScore distribution of in TCGA validation set of are shown in Fig. 5a. It showed that the expression of 5 genes $(A K 4, H K 2$, $P 4 H A 1, T G F B I$ and $V E G F A$ ) increases as the risk value increases, which were consistent with those of the TCGA training set. The ROC analysis of RiskScore classification showed that the AUC was greater than 0.7 at 1,3 and 5 years (Fig. 5b). Based on the calculated median of the RiskScore value, the samples were divided into either a high or low risk group and survival curves were plotted for the two groups. The two groups show a statistically significant difference with regard to prognosis, that is, the established prognosis model can significantly distinguish between risks of the different groups in the test set (Fig. 5c).

RiskScore distribution of all TCGA dataset are shown in Additional file 7: Fig. S3a. We found that $A K 4, H K 2$, P4HA1, TGFBI and VEGFA were associated with high gene expressions and a high risk related factor, results which were consistent with TCGA training set performance findings. The RiskScore classification efficiency of prognosis prediction for 1,3 , and 5 years was analyzed (Additional file 7: Fig. S3b). Based on the calculated median of RiskScore, samples were divided into high and low risk groups and a statistically significantly prognostic difference was present between two groups (Additional file 7: Fig. S3c).

Similarly, for the external validation dataset, GSE44001, the same coefficient as used for the gene in the training set model was used to calculate the RiskScore of each
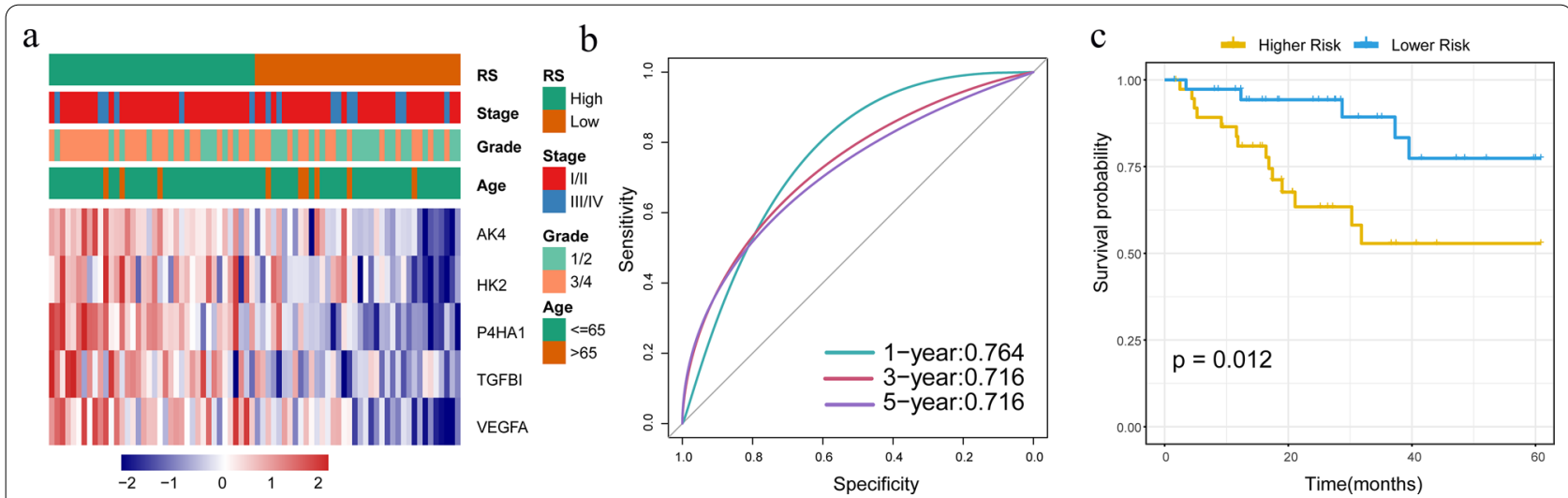

Fig. 5 a Verification of expressions and clinical characteristics of the five prognostic genes in the high and low risk groups. b ROC curves and AUC of RiskScore classifications. c RiskScore in the validation set of the KM survival curve distribution 
sample,the RiskScore grouping is presented in Additional file 8: Fig. S4a. The R software package, pROC, was used to perform the ROC analysis of riskScore prognostic classification for 1, 3 and 5 years (Additional file 8: Fig. S4b). As described above, samples were divided into high and low risk groups and survival curves were drawn. A statistically significant difference in survival was obtained between the two groups as shown in Additional file 8: Fig. S4c. These findings provide further evidence indicating that the model can not only be applied to internal, but also external data sets.

\section{Prognostic analysis of risk models and clinical features}

We further performed correlation analysis with the 5-gene signature and clinical factors, it found that the box diagram, as presented in Fig. 6a, displays the distribution of RiskScore in Grade 3/4 and Grade 1/2 samples, and indicates that the Risk score of Grade 3/4 is significantly greater than that of the Grade 1/2 Risk score. The clinical subtypes, including Grade (1/2), Grade (3/4), Stages $(\mathrm{I} / \mathrm{II})$, Stages $(\mathrm{III} / \mathrm{IV})$, Age $(>65)$ and Age $(\leq 65)$ were grouped based on the xpressions of Risk score, and differences in prognosis between high and low risk groups

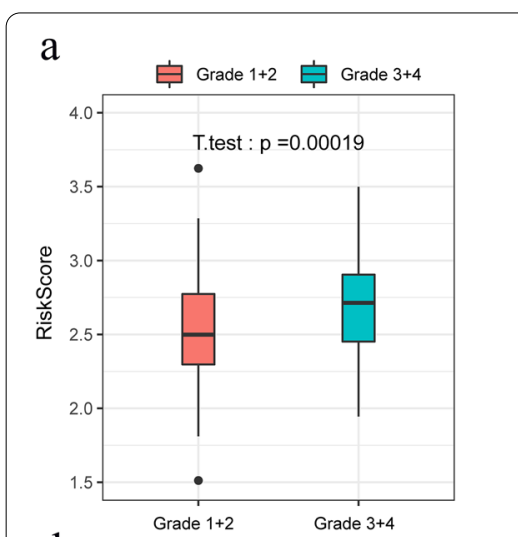

d
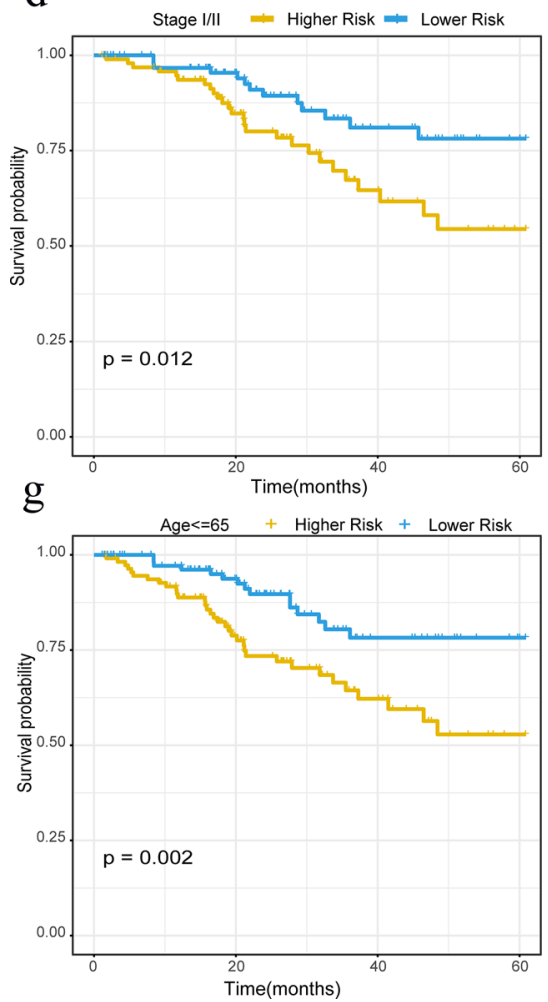
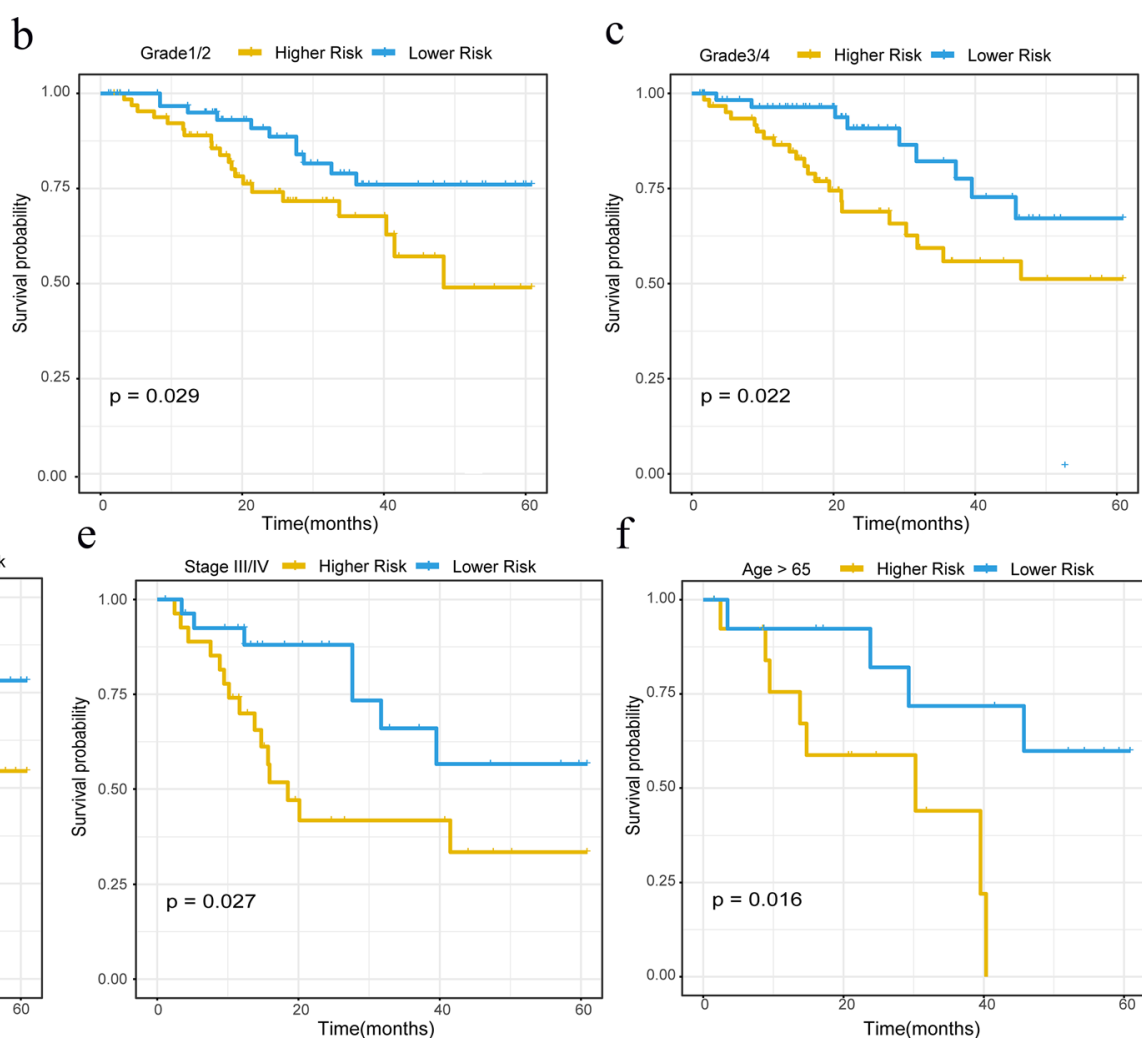

f

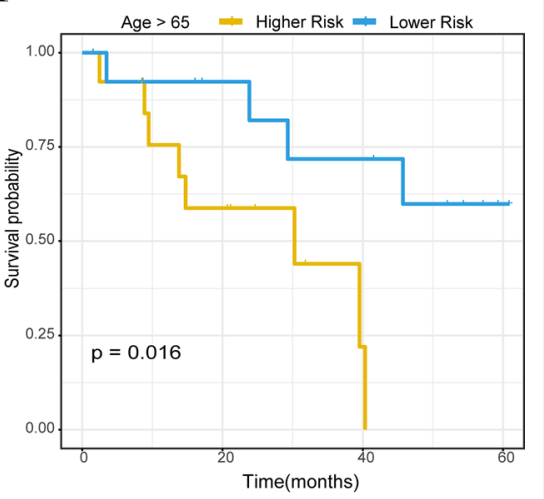

Fig. 6 a Boxplot for Grade 1/2 and Grade 3/4 risk score. b-g Survival curves of clinical subtypes as based on Riskscore 
were assessed. With this analysis, low risk scores were significantly associated with a good prognosis (Fig. 6b-g).

\section{Enrichment analysis based on RiskScore}

The KEGG and GO (BP, MF) (BP: Biological process. MF: Molecular function) items enriched within the high and low risk groups, as based on RiskScore, are shown as barplots. The specific enrichment analysis results are shown in Additional file 9: S5.csv with the KEGG pathway, including the PI3K-Akt signaling pathway, MAPK signaling pathway, Focal adhesion, TGF-beta signaling pathway and ECM-receptor interaction (Additional file 10: Fig. S5a-e).

\section{Univariate and multivariate cox analysis and construction of the nomogram}

The univariate, multivariate analyses and a nomogram model was constructed to evaluate the prediction accuracy of the model. Univariate and multivariate COX regressions were used to analyze the relationship between RiskScores and other variables and clinical prognosis of patients as summarized in Fig. 7. Results from both univariate and multivariate COX analysis revealed that Age $(\mathrm{HR}>1, \mathrm{p}<0.05)$, Stage $(\mathrm{HR}>1, \mathrm{p}<0.01)$ and Risk Score $(H R>1, p<0.05)$ were correlated with prognosis of patients. Accordingly, these factors could be used as independent risk factors for the prognosis of patients with cervical cancer.

Based on the results obtained from the univariate and multivariate cox analysis, the age, Stage and RiskScore of clinical features were combined to construct a nomogram model (Fig. 8a). From this model, RiskScore features were found to exert the greatest impact on survival prediction rates, indicating that this risk model, as based on these 5 genes, can better predict the prognosis of cervical cancer. Further, a calibration curve was used to evaluate the prediction accuracy of the model, as shown in Fig. 8b. It can be seen that the predicted calibration curves of the three calibration points at 1,3 and 5 years have a high degree of coincidence with the standard curves, indicating that the model demonstrates a good level of prediction performance. In addition, we also used DCA to evaluate the reliability of the model, as shown in Fig. 8c. This analysis reveals that the benefits of RiskScore and Nomogram are significantly greater than those of the extreme curve, where the Nomogram is greater than RiskScore and $\mathrm{T}$
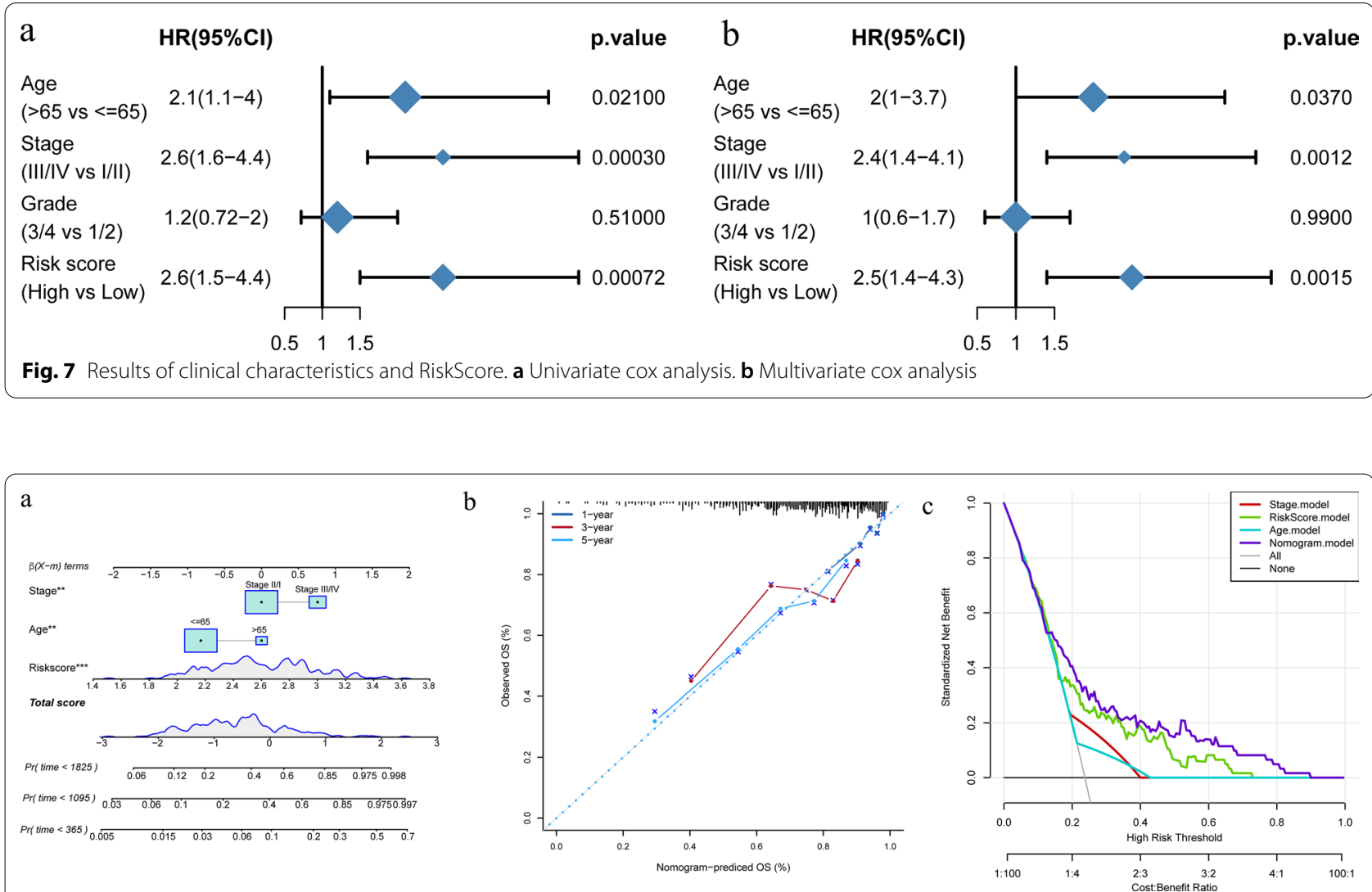

Fig. 8 a Nomogram model for Stage, Riskscore and Age combinations. b 1, 3 and 5-year calibration curves of the Nomogram. c DCA of the Nomogram model 
Stage is close to the extreme curve. Such findings indicate that RiskScore and Nomogram possess a good degree of reliability. The above results demonstrate that a line graph with multiple clinical variables can better predict OS than single indicator.

\section{The AK4, HK2, P4HA1, TGFBI and VEGFA expression are up-regulated in cervical cancer tissue}

Furthermore, to verify the accuracy of the 5-gene signature, we examined the expression of the signature genes (AK4, HK2, P4HA1, TGFBI and VEGFA) in clinical samples from 10 cervical cancer patients by qPCR and IHC analysis. The results obtained with RT-PCR (Fig. 9a) and immunohistochemistry (Fig. 0.9b) assays both showed that AK4, HK2, P4HA1, TGFBI and VEGFA were all highly expressed in these cervical cancer tissue samples. Clinical details of these 10 patients are contained in Additional file 12: S7.xls.

\section{The biological function of signature genes in cervical cancer cells}

To investigate the biological function of AK4, HK2, P4HA1, TGFBI and VEGFA in cervical cancer cells, transwell and colony formation assays were performed. The transwell assays were used to determine the invasion and migration ability of cervical cancer cells, and the colony formation assay was performed to detect the proliferation ability of cells. The colony formation and transwell assays results showed that reduced $A K 4, H K 2$, P4HA1, $T G F B I$ and $V E G F A$ expression, which significantly inhibited proliferation (Fig. 10a), migration (Fig. 10b) and invasion (Fig. 10c) ability of cervical cancer cells.

\section{Discussion}

Cervical cancer is characterized as having a high incidence and mortality rate, particularly in low and middle economic populations of patients. Therefore, it is urgent to determine new prognostic indicators in order to more accurately predict the prognosis of patients with cervical cancer. While there have been many studies directed toward examining the relationship between hypoxia and tumor formation, the relationship between hypoxia and prognosis of patients with cervical cancer remain quite limited. In this study, the hypoxia-related gene signatures was used to predict the prognosis of patients with cervical cancer and demonstrate that this approach achieves good prediction results.

Specifically, we developed a 5-gene signature prognostic risk model and provide a verification of its validity as demonstrated in both train and test data sets. Based on the results of survival analysis, ROC and nomogram, we believe that this constructed risk factor model is quite robust. In addition, in each clinical subtype of TCGA, this risk factor model effectively predicted the risk of cervical cancer. For the first time, we have established a prognostic prediction model based on hypoxia genes.

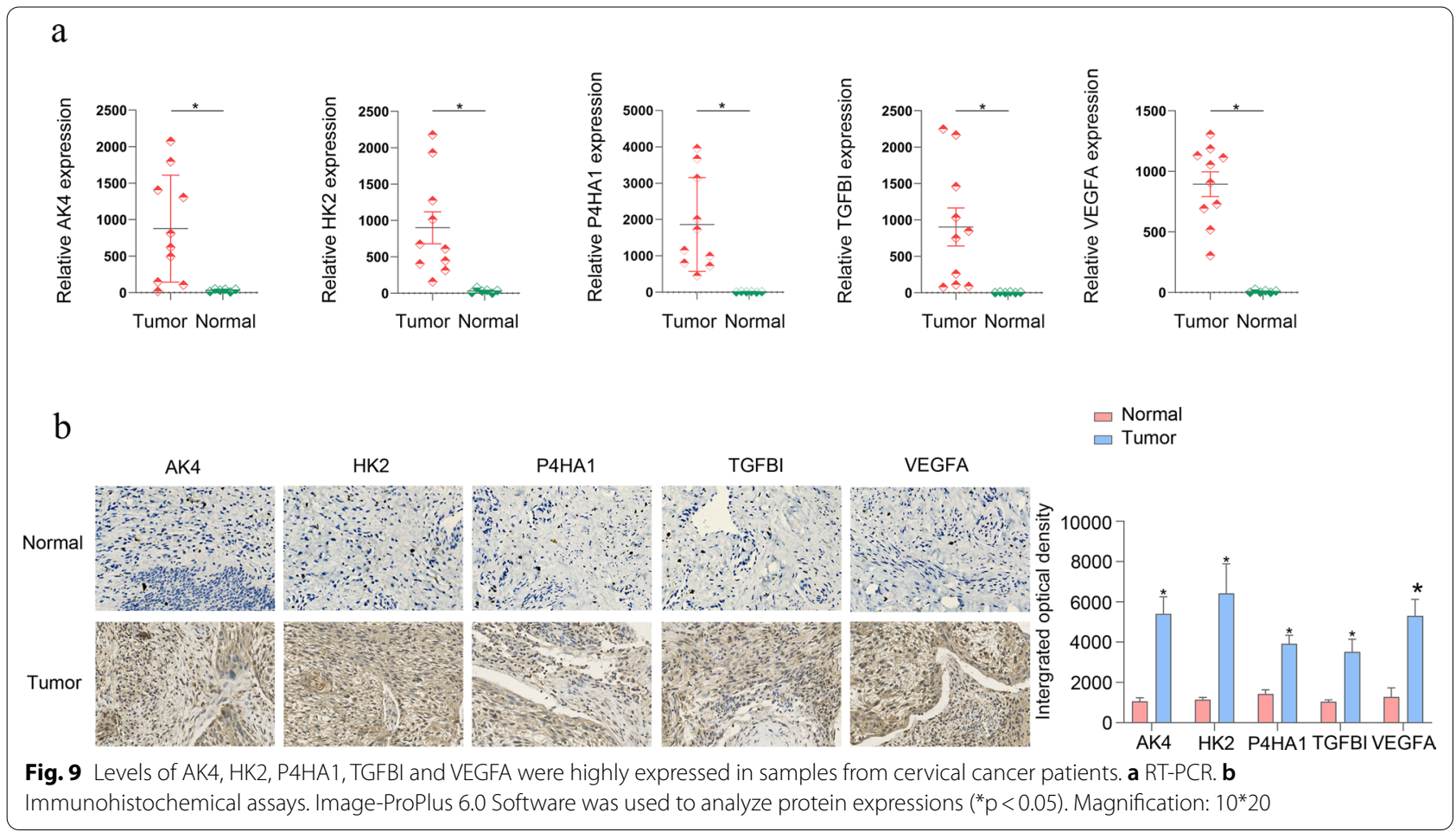




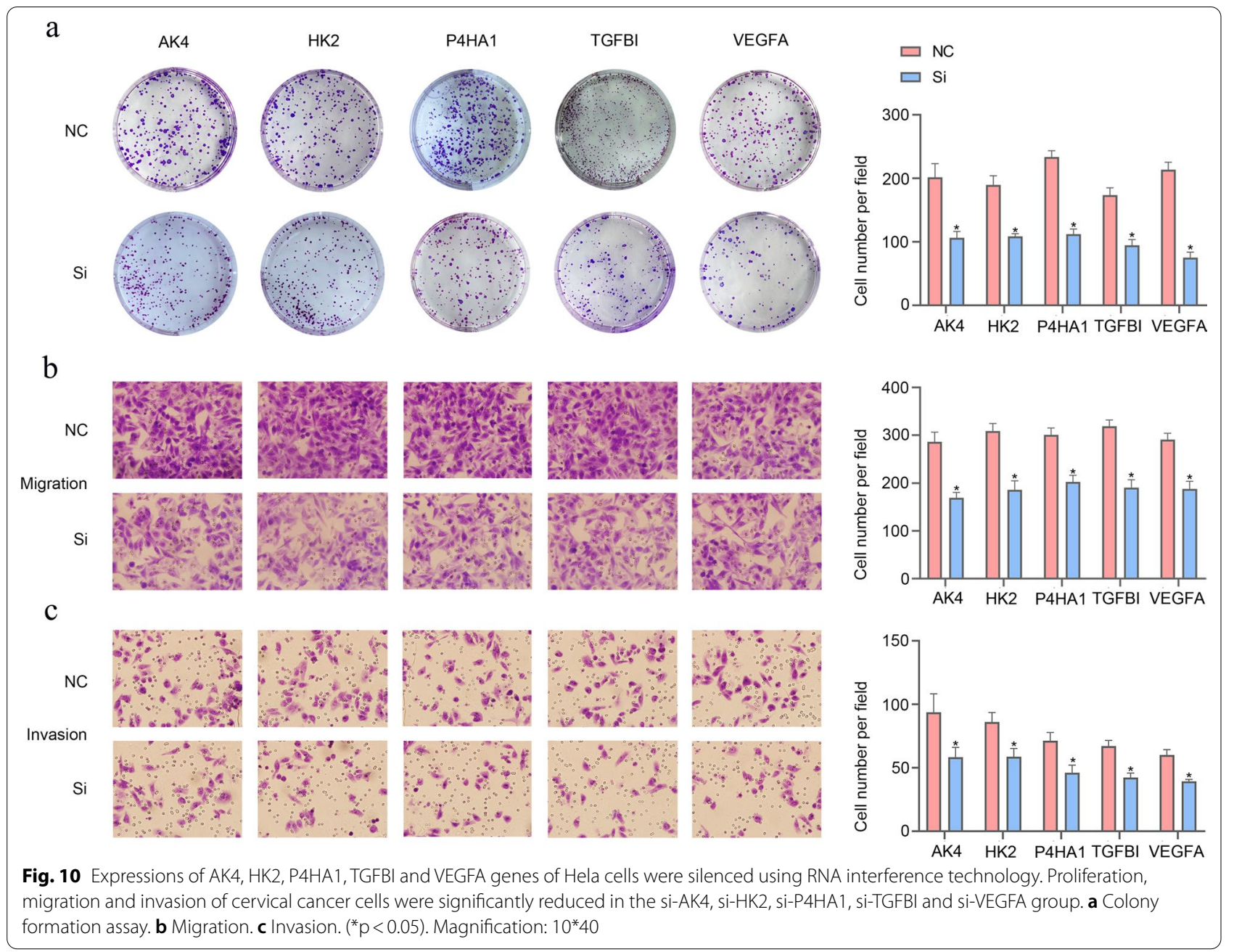

Moreover, the results of qPCR and immunohistochemistry staining assay revealed that the expression of $A K 4$, HK2, P4HA1, TGFBI and VEGFA is high in cervical cancer. The functional study shown that expression of $A K 4$, HK2, P4HA1, TGFBI and VEGFA can regulate the proliferation, migration, and invasion ability of cervical cancer cells. Among them, AK4, P4HA1 and TGFBI were first confirmed as oncogene in cervical cancer. In our study, taken together, we believe that this model can be used to evaluate the prognostic risk in cervical cancer patients. And our model is better than other cervical cancer models [23, 24].

In our analysis, the high expression and high risk of these five hypoxia-related genes, $A K 4, H K 2$, P4HA1, $T G F B I$ and $V E G F A$, were identified as risk factors. Adenylate kinase $4(A K 4)$ is a member of the adenosine kinase family and has been found to play an important role in malignant tumors and anti-tumor therapy. Results from recent studies have shown that high expressions of $A K 4$ promote lung cancer metastasis by enhancing
HIF-1 $\alpha$ stability and EMT under conditions of hypoxia [25]. High expressions of $A K 4$ also promote cell proliferation and invasion in ovarian cancer and HER2 positive breast and esophageal cancers [26-28]. Hexokinase 2 $(H K 2)$ is a rate-limiting enzyme in the glycolysis pathway. In addition to the catalytic activity of this enzyme, $H K 2$ can also antagonize apoptosis within the mitochondrial pathway, which plays an important role in the invasion and metastasis of malignant tumors. It has been reported that $\mathrm{B} 7-\mathrm{H} 3$ promotes aerobic glycolysis and increases chemoresistance in colorectal cancer cells through the upregulation of $H K 2$ [29]. Silencing $H K 2$ in human hepatocellular carcinoma cells inhibits tumorigenesis and increases cell death, and $H K 2$ silencing can synergistically inhibit tumor growth with sorafenib [30]. In colorectal cancer, $P L K 3$ inhibits glucose metabolism by targeting HSP90/STAT3/HK2 signal transduction. Under conditions of $P L K 3$ overexpression, expression levels of $H K 2$ decrease, while silencing $P L K 3$ increases the expression of $H K 2$ in tumor cells. $H K 2$ silencing can inhibit the 
growth of colorectal cancer cells [31]. Proline 4-hydroxylase subunit $\alpha-1$ (P4HA1) is associated with a variety of malignant tumor development pathways, such as EMT, angiogenesis, invasion, inflammation, tumor metabolism and glycolysis pathways [32]. Findings from recent studies have shown that P4HA1 is up-regulated in lung, breast and head/neck cancer tissues, and high expression levels of P4HA1 are significantly correlated with the clinical characteristics of these cancers. The clinical prognosis of patients with high expressions of P4HA1 is poor [33]. In melanoma, depletion of P4HA1 reduces cell adhesion, invasion and in vitro survival, and in xenotransplantation models, knockdown of P4HA1 reduces the invasion of melanoma in vivo and the deposition of collagen in interstitial ECM and tumor vascular basement membranes. Such results indicate that $P 4 H A 1$ can serve as a potential biomarker for poor prognosis of primary melanoma [34]. Upregulation of P4HA1 promotes cell migration and invasion in glioblastoma and head/neck squamous cell carcinoma and, in this way, provides a biomarker for poor prognosis in patients with high expressions of P4HA1 $[35,36]$. As an extracellular matrix protein, TGFBI is closely related to the development of different malignant tumors. In glioma, the median survival time for patients with a high expression of $T G F B I$ was significantly shorter than that of patients showing low expressions of TGFBI [37]. An up-regulation of TGFBI can promote the occurrence and metastasis of breast cancer, increase tumor angiogenesis and increase hypoxia, and TGFBI overexpression promotes oral squamous cell carcinoma. Knockout of TGFBI inhibits the proliferation and metastasis of oral squamous cell carcinoma in vivo. Accordingly, low levels of TGFBI expression can predict a better prognosis [38], while patients with TGFBI overexpression have a poor prognosis [39]. Vascular endothelial growth factor A (VEGFA) plays an important role in tumor angiogenesis. VEGFA may be a prognostic gene in clear cell renal cell carcinoma, as significant increases in VEGFA are associated with a poor prognosis [40]. Upregulation of VEGFA also indicates a poor prognosis for lung adenocarcinoma and oral squamous cell carcinoma, suggesting that VEGFA represents a valuable prognostic biomarker $[41,42]$.

The new and important findings of this study are the identification of a prognostic 5-gene signature with a relatively high AUC in the training and test dataset, which can then predict 1,3 and 5-year survival rates. We also established a series of clinical variables of the nomogram model and verified these predicted genes in a series of experiments, which then substantiated the reliability of the prediction. In the future, genetic diagnosis and treatment will become more effective means. The expression of 5-gene signature in cervical cancer tissues will be detected by qPCR, and the model genes will be converted into risk scores for predicting the prognosis of patients, which is significance of clinical application. The limitation of this study is that some data lacked clinical follow-up information and further genetic and experimental studies along with experimental verification will be required with larger samples. Moreover, direct clinical application tests of this prognosis model will need to be conducted.

In summary, our study developed a 5-gene signature prognostic hierarchical system based on the hypoxic pathway of cervical cancer. This protocol shows an effective AUC in the training and independent test set, and serves as a model which is independent of clinical characteristics. Further, in order to verify the signature, TCGA and GSE44001 datasets was used to test, and finally got a good risk prediction effect in those datasets. We also conducted experimental verifications on these five genes and found that the expression of $A K 4$, $H K 2, P 4 H A 1, T G F B I$ and VEGFA were high in cervical cancer tissues. Moreover, silencing these genes inhibited the proliferation, migration and invasion ability of cervical cancer cells as demonstrated in vitro. Therefore, these results suggesting that the signature could potentially be used to evaluate the prognostic risk of cervical cancer patients, and provide potential targets for the treatment of cervical cancer patients.

\section{Abbreviations \\ TCGA: The Cancer Genome Atlas; HPV: Human papilloma virus; CT: Computer Tomography; MRI: Magnetic Resonance Imaging; PET-CT: Positron emis- sion tomography computer Tomography; EMT: Epithelial-mesenchymal transition; GLUTs: Glucose transporters; GEO: Gene Expression Omnibus; OS: Overall survival; CDF: Cummulative distribution function; GO: Gene Ontology; KEGG: Kyoto Encyclopedia of Genes and Genomes; ROC: Receiver operating characteristic; DCA: Decision Curve Analysis; IHC: Immunohistochemistry; DEGs: Differential expressed genes; AUC: Area Under the Curve; BP: Biological process; MF: Molecular function.}

\section{Supplementary Information}

The online version contains supplementary material available at https://doi. org/10.1186/s12935-021-02050-3.

Additional file 1. The cox analysis results of all hypoxia pathway-related genes.

Additional file 2: Fig. S1. The Cluster2 vs Cluster1 differential analysis. a gene volcano map. b gene heat map.

Additional file 3. Detailed differentially expressed genes.

Additional file 4: Fig. S2. Annotations (maps) of differentially expressed genes between Cluster2 and Cluster1. a KEGG. b BP. c MF.

Additional file 5. Detailed analysis results of KEGG pathway.

Additional file 6. Detailed analysis results of GO functional enrichment analyses.

Additional file 7: Fig. S3. a Expressions and clinical features of five prognostic genes in the high and low risk groups with regard to TCGA. 
b ROC curves and AUC of RiskScore classifications. c KM survival curve distribution of RiskScore in all TCGA sets.

Additional file 8: Fig. S4. a Expression heat maps of five model genes in the high and low risk groups with regard to GSE44001. b ROC curves and AUC of RiskScore classifications. c KM survival curve distribution of RiskScore in GSE44001.

Additional file 9. Specific results of enrichment analysis based on RiskScore.

Additional file 10: Fig. S5. a-e KEGG enrichment analysis results for the high and low risk groups.

Additional file 11. Sequences of upstream and downstream primers.

Additional file 12. Clinical details of these cervical cancer 10 patients.

\section{Acknowledgements}

Not applicable.

\section{Authors' contributions}

LZ and YY designed the research, analyzed the data, and wrote the manuscript; LZ, YY and YLL performed the experiments; LZ collected the tissue samples; $L Z$ and RQQ interpreted the data. All authors read and approved the final manuscript.

\section{Funding}

This work was supported by the National Natural Science Foundation of China (No. 81803148) and Natural Science Foundation of Liaoning Province (No.2019-KF-01-07).

\section{Availability of data and materials}

The datasets used and/or analyzed during the current study are available from the corresponding author on reasonable request.

\section{Declarations}

\section{Ethics approval and consent to participate}

Informed consent was obtained from all patients and the research was approved by the Ethics Committee of First Hospital of China Medical University (2016-207-4).

\section{Consent for publication}

Not applicable.

\section{Competing interests}

The authors declare no competing interests.

Received: 10 April 2021 Accepted: 24 June 2021

Published online: 03 July 2021

\section{References}

1. Bray F, Ferlay J, Soerjomataram I, Siegel RL, Torre LA, Jemal A. Global cancer statistics 2018: GLOBOCAN estimates of incidence and mortality worldwide for 36 cancers in 185 countries. CA Cancer J Clin. 2018:68:394-424.

2. Cancer: Disease Control Priorities, Third Edition (Volume 3). 2015. Washington (DC). The International Bank for Reconstruction and Development / The World Bank.

3. Bruni L, Diaz M, Barrionuevo-Rosas L, Herrero R, Bray F, Bosch FX, et al. Global estimates of human papillomavirus vaccination coverage by region and income level: a pooled analysis. Lancet Glob Health. 2016:4:e453-63.

4. Sadri Nahand J, Moghoofei M, Salmaninejad A, Bahmanpour Z, Karimzadeh $M$, Nasiri M, et al. Pathogenic role of exosomes and microRNAs in HPV-mediated inflammation and cervical cancer: a review. Int J Cancer. 2020;146:305-20
5. Scheidler J, Hricak H, Yu KK, Subak L, Segal MR. Radiological evaluation of lymph node metastases in patients with cervical cancer : A meta-analysis. JAMA. 1997:278:1096-101.

6. Chao A, Ho KC, Wang CC, Cheng HH, Lin G, Yen TC, et al. Positron emission tomography in evaluating the feasibility of curative intent in cervical cancer patients with limited distant lymph node metastases. Gynecol Oncol. 2008;110:172-8.

7. Nogami Y, Banno K, Irie H, lida M, Kisu I, Masugi Y, et al. The efficacy of preoperative positron emission tomography-computed tomography (PET-CT) for detection of lymph node metastasis in cervical and endometrial cancer: clinical and pathological factors influencing it. Jpn J Clin Oncol. 2015;45:26-34.

8. Buchberger W, Oberaigner W, Kremser C, Gautsch K, Siebert U. Non-mass enhancement in breast mri: characterization with BI-RADS descriptors and ADC values. SciMedicine J. 2021;3:77-87.

9. Pourhanifeh MH, Darvish M, Tabatabaeian J, Fard MR, Mottaghi R, Azadchehr MJ, et al. Therapeutic role of curcumin and its novel formulations in gynecological cancers. J Ovarian Res. 2020;13:130.

10. Huang C, Lin X, He J, Liu N. Enrichment and detection method for the prognostic value of circulating tumor cells in ovarian cancer: a metaanalysis. Gynecol Oncol. 2021;161:613-20.

11. Nahand JS, Vandchali NR, Darabi H, Doroudian M, Banafshe HR, Moghoofei $\mathrm{M}$, et al. Exosomal microRNAs: novel players in cervical cancer. Epigenomics. 2020;12:1651-60.

12. Hashemipour M, Boroumand H, Mollazadeh S, Tajiknia V, Nourollahzadeh Z, Rohani Borj M, et al. Exosomal microRNAs and exosomal long noncoding RNAs in gynecologic cancers. Gynecol Oncol. 2021;161:314-27.

13. Razavi ZS, Tajiknia V, Majidi S, Ghandali M, Mirzaei HR, Rahimian N, et al. Gynecologic cancers and non-coding RNAs: epigenetic regulators with emerging roles. Crit Rev Oncol Hematol. 2021;157:103192.

14. Kosvyra A, Maramis C, Chouvarda I. Developing an integrated genomic profile for cancer patients with the use of NGS data. Emerg Sci J. 2019;3:157-67.

15. Tirpe AA, Gulei D, Ciortea SM, Crivii C, Berindan-Neagoe I. Hypoxia: overview on hypoxia-mediated mechanisms with a focus on the role of HIF genes. Int J Mol Sci. 2019;20:6140

16. Jing $X$, Yang F, Shao C, Wei $K$, Xie M, Shen $H$, et al. Role of hypoxia in cancer therapy by regulating the tumor microenvironment. Mol Cancer. 2019;18:157.

17. Lamouille S, Xu J, Derynck R. Molecular mechanisms of epithelial-mesenchymal transition. Nat Rev Mol Cell Biol. 2014;15:178-96.

18. Liberti MV, Locasale JW. The Warburg effect: how does it benefit cancer cells. Trends Biochem Sci. 2016:41:211-8.

19. Yang Y, Qu A, Wu Q, Zhang X, Wang L, Li C, et al. Prognostic value of a hypoxia-related microRNA signature in patients with colorectal cancer. Aging (Albany NY). 2020;12:35-52.

20. Ren S, Liu J, Feng Y, Li Z, He L, Li L, et al. Knockdown of circDENND4C inhibits glycolysis, migration and invasion by up-regulating miR-200b/c in breast cancer under hypoxia. J Exp Clin Cancer Res. 2019;38:388.

21. Brooks JM, Menezes AN, Ibrahim M, Archer L, Lal N, Bagnall CJ, et al. Development and validation of a combined hypoxia and immune prognostic classifier for head and neck cancer. Clin Cancer Res. 2019:25:5315-28

22. Zare $\mathrm{H}$. Effects of salvia officinalis extract on the breast cancer cell line. SciMedicine J. 2019;1:25-9.

23. Chen Q, Qiu B, Zeng X, Hu L, Huang D, Chen K, et al. Identification of a tumor microenvironment-related gene signature to improve the prediction of cervical cancer prognosis. Cancer Cell Int. 2021;21:182.

24. Pan J, Xu L, Pan H. Development and validation of an m6A RNA methylation regulator-based signature for prognostic prediction in cervical squamous cell carcinoma. Front Oncol. 2020;10:1444.

25. Jan YH, Lai TC, Yang CJ, Lin YF, Huang MS, Hsiao M. Adenylate kinase 4 modulates oxidative stress and stabilizes HIF-1 a to drive lung adenocarcinoma metastasis. J Hematol Oncol. 2019;12:12.

26. Tan H, Wu C, Huang B, Jin L, Jiang X. MiR-3666 serves as a tumor suppressor in ovarian carcinoma by down-regulating AK4 via targeting STAT3. Cancer Biomark. 2020;4:1-9.

27. Zhang J, Yin YT, Wu CH, Qiu RL, Jiang WJ, Deng XG, et al. AK4 promotes the progression of HER2-positive breast cancer by facilitating cell proliferation and invasion. Dis Markers. 2019;2019:8186091. 
28. Zang C, Zhao F, Hua L, Pu Y. The miR-199a-3p regulates the radioresistance of esophageal cancer cells via targeting the AK4 gene. Cancer Cell Int. 2018;18:186.

29. Shi T, Ma Y, Cao L, Zhan S, Xu Y, Fu F, et al. B7-H3 promotes aerobic glycolysis and chemoresistance in colorectal cancer cells by regulating HK2. Cell Death Dis. 2019;10:308.

30. DeWaal D, Nogueira V, Terry AR, Patra KC, Jeon SM, Guzman G, et al. Hexokinase-2 depletion inhibits glycolysis and induces oxidative phosphorylation in hepatocellular carcinoma and sensitizes to metformin. Nat Commun. 2018;9:446.

31. Ou B, Sun H, Zhao J, Xu Z, Liu Y, Feng H, et al. Polo-like kinase 3 inhibits glucose metabolism in colorectal cancer by targeting HSP90/STAT3/HK2 signaling. J Exp Clin Cancer Res. 2019;38:426.

32. Balamurugan K. HIF-1 at the crossroads of hypoxia, inflammation, and cancer. Int J Cancer. 2016;138:1058-66.

33. Li M, Wu F, Zheng $Q$, Wu Y, Wu Y. Identification of potential diagnostic and prognostic values of P4HA1 expression in lung cancer, breast cancer, and head and neck cancer. DNA Cell Biol. 2020;39:909-17.

34. Eriksson J, Le Joncour V, Jahkola T, Juteau S, Laakkonen P, Saksela O, et al. Prolyl 4-hydroxylase subunit alpha 1 (P4HA1) is a biomarker of poor prognosis in primary melanomas, and its depletion inhibits melanoma cell invasion and disrupts tumor blood vessel walls. Mol Oncol. 2020;14:742-62

35. Zhu X, Liu S, Yang X, Wang W, Shao W, Ji T. P4HA1 as an unfavorable prognostic marker promotes cell migration and invasion of glioblastoma via inducing EMT process under hypoxia microenvironment. Am J Cancer Res. 2021:11:590-617.
36. Wu ZH, Zhong Y, Zhou T, Xiao HJ. miRNA biomarkers for predicting overall survival outcomes for head and neck squamous cell carcinoma. Genomics. 2021;113:135-41.

37. Pan YB, Zhang CH, Wang SQ, Ai PH, Chen $\mathrm{K}$, Zhu L, et al. Transforming growth factor beta induced (TGFBI) is a potential signature gene for mesenchymal subtype high-grade glioma. J Neurooncol. 2018;137:395-407.

38. Fico F, Santamaria-Martínez A. TGFBI modulates tumour hypoxia and promotes breast cancer metastasis. Mol Oncol. 2020;14:3198-210.

39. Wang BJ, Chi KP, Shen RL, Zheng SW, Guo Y, Li JF, et al. TGFBI promotes tumor growth and is associated with poor prognosis in oral squamous cell carcinoma. J Cancer. 2019;10:4902-12.

40. Wang X, Zhang J, Wang Y, Tu M, Wang Y, Shi G. Upregulated VEGFA and DLL4 act as potential prognostic genes for clear cell renal cell carcinoma. Onco Targets Ther. 2018;11:1697-706.

41. Qin S, Yi M, Jiao D, Li A, Wu K. Distinct roles of VEGFA and ANGPT2 in lung adenocarcinoma and squamous cell carcinoma. J Cancer. 2020;11:153-67.

42. Peterle GT, Maia LL, Trivilin LO, de Oliveira MM, Dos Santos JG, Mendes SO, et al. PAI-1, CAIX, and VEGFA expressions as prognosis markers in oral squamous cell carcinoma. J Oral Pathol Med. 2018;47:566-74.

\section{Publisher's Note}

Springer Nature remains neutral with regard to jurisdictional claims in published maps and institutional affiliations.
Ready to submit your research? Choose BMC and benefit from:

- fast, convenient online submission

- thorough peer review by experienced researchers in your field

- rapid publication on acceptance

- support for research data, including large and complex data types

- gold Open Access which fosters wider collaboration and increased citations

- maximum visibility for your research: over $100 \mathrm{M}$ website views per year

At BMC, research is always in progress.

Learn more biomedcentral.com/submissions 